\title{
The DCMT in Progress
}

\section{Li Zhigang}

Shaanxi Astronomical Observatory P.O. Box 18 , Lintong

Shaanxi, P.R. China

\begin{abstract}
The DCMT (Danish-Chinese Meridian Telescope) is jointly developed by the Shaanxi Astronomical observatory, Nanjing Astronomical Instrument Factory and Copenhagen University Observatory. The instrument was set up at the Shaanxi Astronomical Observatory in 1990 and put in testing operation in the same year. The design of this horizontal meridian circle is very compact and allows direct real-time monitoring of the instrument parameters. We have studied the stability of the instrument and internal refraction in the telescope tube.
\end{abstract}

\section{INTRODUCTION TO THE DCMT}

The DCMT is an automatic horizontal meridian circle which was developed by China and Denmark. The DCMT was installed at the Shaanxi Astronomical Observatory, China, in Apr. 1990 and made testing observations with visual micrometer in the same year. According to the observed results, some modifications and adjustments were done. More regular observations were started from the beginning of 1992. Recently, a new photoelectric micrometer has been manufactured and will be put in real operation. With the new micrometer, the instrument will realize automation.

Fig. 1 shows the principle of the horizontal meridian circle. It consists of two double mirrors $s_{1}, s_{2}$ and $s_{3}, s_{1}$. The telescope is composed of $S_{1}$ and $S_{3}$ and autocollimation is composed of $S_{2}$ and $S_{4}$. The glass circle, micrometer, $S_{1}$ and $s_{2}$ mirrors are fixed together, all of them are named as the main box. The main box is supported by a bearing with a horizontal east-west axis. An angle between $S_{1}$ and $S_{2}$ is defined as the collimation of the instrument. The rotation of $s_{1}$ and $s_{2}$ around the optical axis by the bearing makes a star in the meridian plane be imaged on the focal plane. The $S_{1}$ and $S_{2}$ are glued to each other. They are not rotatable and are fixed to the base frame. A calibration point in the micrometer forms two images.One is the image reflected from $S_{4}$ since the focal length of $S_{4}$ is as long as half of $S_{3}$. Using coordinates of the calibration point and the image, the direction of the optical axis is determined. Another is the image after three reflections on $S_{3}, S_{2}$ and again on $S_{3}$. It can determine the tilt of $s_{1}$.

The DCMT is a new kind of meridian circles. It has the advantages over classical meridian circles:

a. The weakness of the setting with whole tube motion for the

79

I.I. Mueller and B. Kotaczek (eds.),

Developments in Astrometry and Their Impact on Astrophysics and Geodynamics, 79-83.

(C) 1993 IAU. Printed in the Netherlands. 
classical meridian circle is avoided on the DCMT.

b. Real time measurements of the instrumental parameters with the autocollimation.

c. Smaller flexure.

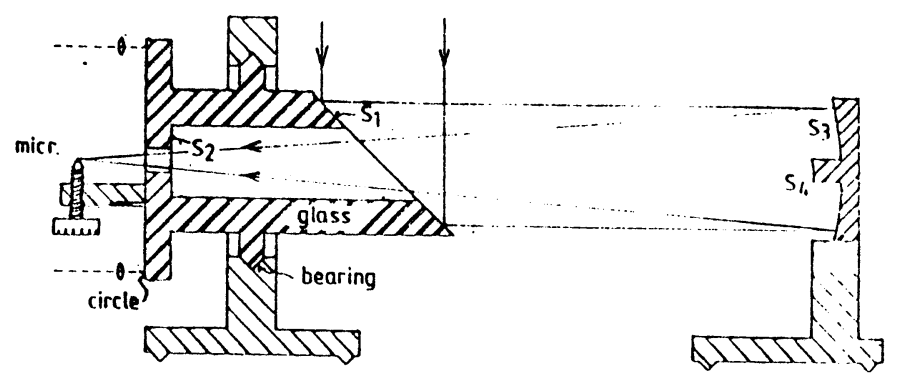

Fig. 1 Optical system of the DCMT

\section{CONTROL SYSTEM}

For convenience of adjustments, our control system is composed of a background computer and several single board computers. Each single board computer performs a special function. All of them are controlled by the background computer. The DCMT control system is showing in Fig.2.

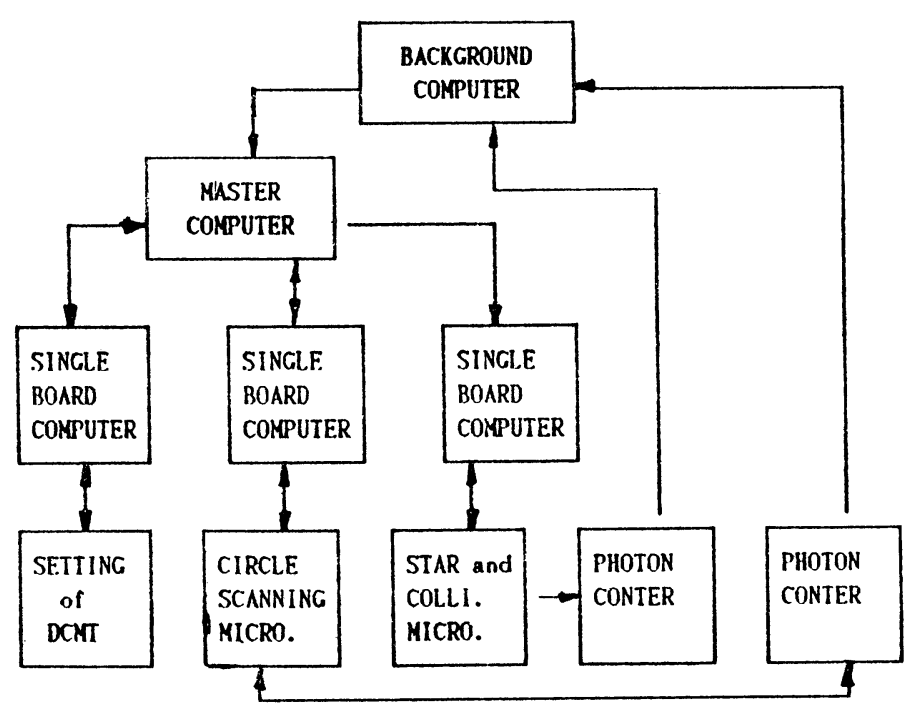

Fig.2 Control System on DCMT 


\section{DETERMINATIONS OF INSTRUMENTAL PARAMETERS}

Since the beginning of 1992 , we have started regular visual observations on the DCMT. The following results from observations were obtained in duration from Jan. to June, 1992 .

\subsection{Stability of the Collimation}

The collimation of the DCMT should not change with the surrounding temperature, since $S_{1}$ and $S_{2}$ mirrors are connected by copper which has a good thermal conductivity. The observation results in the past 7 months show no significant variation with the surrounding temperature (see Fig.3). The jump from winter to summer may have another reason other than due to change of temperature.

\subsection{The Setting System}

The DCMT is driven by a pair of DC motors. The encoder monitors the setting position of the DCMT and send the message to the single board computer. According to the message, the computer will control the DC motors' drive. The accuracy of the setting is about $5 . " 0$ standard error.

\subsection{The Pivot Error}

Since the pivot error of the horizontal meridian circle is determined in real time, we are interested in the repeatabilit. of determinations for the pivot error. A long series of determinations with different temperature and direction of the setting have been done. The repeatability is about 0.06 . Tab. shows the instrumental parameters from determinations.

$\begin{array}{lc}\text { Tab.1. The determined instrumental parameters } \\ \text { Accuracy of the setting } & 5 . " 0 \\ \text { Resolution of the encoder } & 1 . " 2 \\ \text { Variation of colli. with temp. } & 0 . " 3 / \mathrm{K} \\ \text { Repeatability of pivot error } & 0 . " 06 \\ \text { Variation of azimuth with temp. } & 0 . " 18 / \mathrm{K}\end{array}$

\section{THE INTERNAL REFRACTION}

A horizontal meridian circle has the problem of internal refraction in the tube as found in the classical meridian circle, see $\operatorname{Hog}(1984), \operatorname{Hog}$ and Miller(1986) and Hog and Fabricius(1988). The diurnal variation of instrumental constant is mainly from the variation of surrounding temperature (see Yoshizawa, 1986). In the DCMT, the thermal gradient is up to $0.3--0.5 \mathrm{~K} / \mathrm{m}$ in the night and $1.5--2.0 \mathrm{~K} / \mathrm{m}$ in the day. The methods to remove internal 
refraction in the tube were discussed by Hu Ningsheng(1984). Hog(1984) proposed a method of tangential ventilation of the telescope tube to remove the internal refraction which is successfully used on the CAMC. We use the same method on our instrument. Usually, the internal refraction is completely removed in $15 \mathrm{~s}$ after switching on the ventilation. During ventilation perfect diffraction images are obtained, in contrast to the often poor images seen without ventilation. This required a careful design of the ventilation system, especially the nozzles injecting the air tangentially into the tube.

The temperature gradient in the DCMT tube has been measured. The repeatability of the electronic thermometer is with $0.1 \mathrm{~K}$. No horizontal temperature gradient in the south-north direction is found. Only an air temperature gradient in the vertical direction has been found. Since the telescope is horizontal,

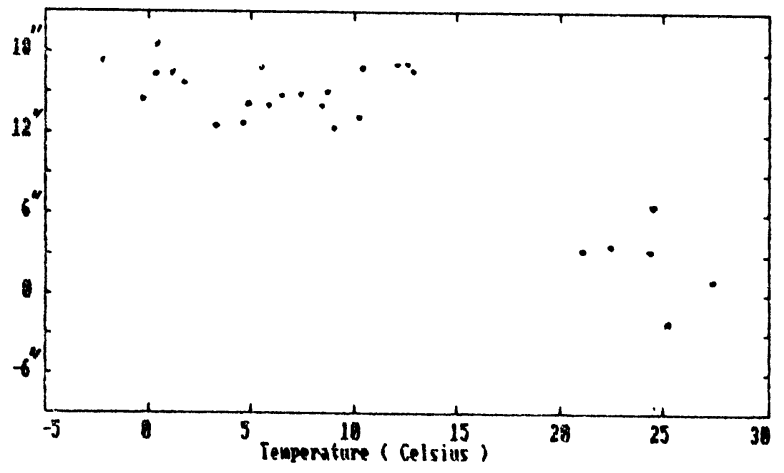

Fig. 3 The variation of the collimation with temperature in the duration from Jan. to June, 1992 .

the stellar image is only moved by the vertically internal. refraction. We have the expressions of effect of the internal. refraction on right ascension $r_{a}$ and declination $r_{\delta}$, respectively,

$$
\begin{aligned}
& r_{a}=b \cdot \cos z \cdot \sec \delta \\
& r_{8}=b \cdot \sin z
\end{aligned}
$$

where $z$ is the zenith distance of the instrument, $b$ is the angular deflection in vertical direction.

The angular deflection for a parallel beam of light travelling perpendicular to a temperature gradient is

$$
b_{1}=0 . " 20 \Delta t \cdot L_{1} / D
$$

For a converging beam of light it is

$$
b_{q}=0 . " 10 \Delta t \cdot L_{q} / D
$$

where $L_{1}$, $L_{2}$ are the lengths of light travelling, $\Delta t$ is the difference of the air temperature in the tube across the aperture D.

For the DCMT, the stellar light will be at first reflected from $S_{1}$ to form a parallel beam and then it will be reflected from $S_{3}$ to form converging beam. So that the angular deflection 
is equal to the sum of $b_{1}$ and $b_{2}$. In our case, $D$ is $260 \mathrm{~mm}$, the focal length $L_{2}$ is $2667 \mathrm{~mm}$, and $L_{1}$ is $2000 \mathrm{~mm}$, which gives $\mathrm{b}=2$. $" 56$.

For the autocollimation image from $S_{1}$, the light beam is converging so that $b=2$." 05 .

We observed both the temperature gradient and the corresponding shift of image and $b \approx 1 . " 7$ was obtained. This is in satisfactory agreement with the theoretical value of 2.05 .

\section{REFERENCES}

Hog,E.: 1984, IAU Symposium, No.109, 533.

Hog,E., Fabricius C.: 1988, Astron. \& Astrophys., 196, 301-312.

Hog, E., Miller J., : 1986, A.J., Vol.92, No.2, 499-503.

Hu Ningsheng : 1984, IAU Symposium, No.109, 469.

Yoshizawa M.: 1986, Diurnal Variation of Instrumental Constant of the Tokyo PMC Ann. Tokyo Astron. Obs., 2nd Series, Vol.21, No.2, 219 . 